\title{
Two-photon interference from remote quantum dots with inhomogeneously broadened linewidths
}

\author{
P. Gold, A. Thoma, S. Maier, S. Reitzenstein, ${ }^{*}$ C. Schneider, ${ }^{\dagger}$ S. Höfling, ${ }^{\ddagger}$ and M. Kamp \\ Technische Physik, Physikalisches Institut and Wilhelm Conrad Röntgen-Research Center for Complex Material Systems, \\ Universität Würzburg, Am Hubland, D-97074 Würzburg, Germany
}

(Received 16 July 2013; revised manuscript received 23 November 2013; published 27 January 2014)

\begin{abstract}
In this paper, we study the influence of quasiresonant and nonresonant excitation on the interference properties of single photons emitted from quantum dots (QDs). The quasiresonant excitation scheme leads to an increase of interference visibility of photons emitted from the same QD to 69\% compared to $12 \%$ for nonresonant excitation. Furthermore, we demonstrate quantum interference of photons emitted from separate QDs which are simultaneously excited into their $p$ shell. We can readily extract a two-photon interference visibility as high as $(39 \pm 2) \%$ for nonpostselected coincidences exceeding the predicted value based on coherence and radiative decay times of the quantum dot emission $(\sim 25 \%)$. We account for this observation by treating the emission of both quantum dots as inhomogeneously broadened ensembles of Fourier-limited photons and observe good congruence between experiment and model.
\end{abstract}

DOI: 10.1103/PhysRevB.89.035313

PACS number(s): 78.67.Hc, 42.50.Dv, 42.50.Ar, 42.50.St

\section{INTRODUCTION}

Single, indistinguishable photons are at the heart of applications in quantum communication, quantum networks, and linear optical quantum computing [1-3]. Promising sources that emit photons of these characteristics are semiconductor quantum dots (QDs). They combine a very good suppression of multiple photon emission events [4], yield the capability to emit indistinguishable photons [5], and can be implemented into nanostructure waveguides and microcavities for high extraction efficiencies [6-9] and enhancement of emission through light-matter coupling effects [10]. However, the generation of indistinguishable photons from separate QDs still is a challenging, yet very appealing task, as it is a crucial part of single-photon quantum repeater networks, entanglement of remote stationary quantum bits (Qbits), and advanced optical teleportation schemes [11-15]. A high degree of indistinguishability can only be achieved if the single-photon wave-packet overlap in space, energy, time, and polarization is sufficiently large. While polarization and space overlap can be implemented routinely in optical setups, it is significantly more demanding to achieve energy and time overlap since they strongly depend on the intrinsic emission properties of the individual QDs.

Recently, two-photon interference (TPI) as a signature of indistinguishable emission from separate quantum dots was shown in a Hong-Ou-Mandel (HOM) experiment by Patel et al. (Ref. [16]) recorded under dc current injection and continuous wave (cw) excitation conditions, by Flagg et al. (Ref. [17]) under pulsed optical excitation, and by Konthasinghe et al. (Ref. [18]) in cw resonant $s$-shell excitation. The postselected visibilities of the TPI in the two nonresonant experiments were $33 \%$ [16], 47\% [17], and 44\% [18] for the resonant $s$-shell excitation scheme. More important in terms of applications is the

\footnotetext{
*Present address: Institut für Festkörperphysik, Technische Universität Berlin, Hardenbergstraße 36, D-10623 Berlin, Germany.

${ }^{\dagger}$ christian.schneider@physik.uni-wuerzburg.de

${ }_{\ddagger}^{\ddagger}$ Present address: SUPA, School of Physics and Astronomy, University of St Andrews, St Andrews, KY16 9SS, United Kingdom.
}

nonpostselected value, which can only be determined in pulsed excitation. In Ref. [17] the nonresonant excitation scheme most likely imposed limitations on the maximum achievable interference visibility of only $18.1 \%$ [17]. It is worth noting that recent interference experiments performed under resonant $s$-shell excitation [15] indeed resulted in markedly increased TPI visibilities (up to 82\%), however, by applying time filtering of the emission to erase the detrimental influence of the resonant laser on the photon statistics. In this experiment the high visibilities are justified by treating the individual QD emission lines as an inhomogeneous broadened signal.

In this paper, we study and probe the TPI from two QDs by exciting them either quasiresonantly into their $p$-shell or nonresonantly into the wetting layer with a picosecond-pulsed laser. Both QDs behave as near-ideal single-photon sources in such an excitation scheme, without the necessity of time filtering to erase the excitation laser. By applying quasiresonant excitation, we could strongly increase the HOM interference contrast from consecutive photons emitted from the same QD as compared to the wetting layer excitation. We demonstrate that it is a direct result of the suppression of carrier recapturing processes which spoil the nonpostselective interference. In a HOM interference experiment performed on photons emitted from two separate QDs, we can readily observe TPI visibilities of $v=(39 \pm 2) \%$ for nonpostselected coincidences, without the application of any time filtering. This value significantly exceeds the expected value of $\sim 25 \%$, typically inferred from the ratio of spontaneous emission lifetime versus coherence time. We can nevertheless justify such large visibilities by treating both QDs as an inhomogeneously broadened ensemble (over time) of Fourier-limited emitters which yields $v_{\max , \text { inhom }}=$ $(36.4 \pm 1.5) \%$ in good quantitative agreement with the value obtained directly from the HOM interference experiment.

\section{SETUP AND SAMPLE CHARACTERISTICS}

Our sample consists of a low-density single layer of $\mathrm{In}(\mathrm{Ga})$ As QDs which is embedded in an asymmetric planar cavity. The resonator consists of five (18) quarter-wavelength AlAs/GaAs mirror pairs in the top (bottom) distributed Bragg reflector and a 1 - $\lambda$-thick central cavity layer. The vertical 


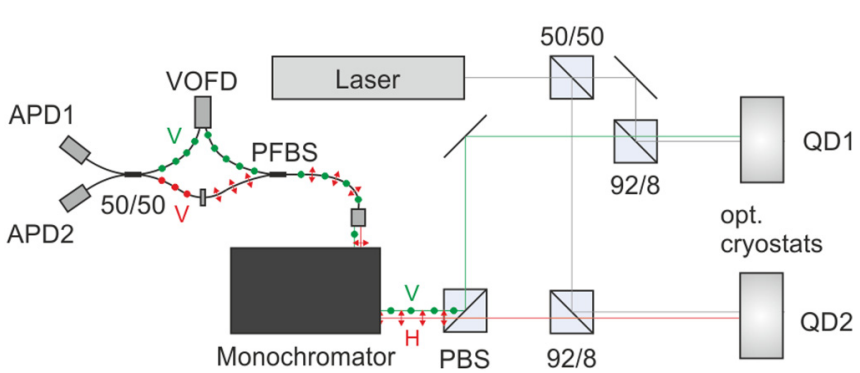

FIG. 1. (Color online) Schematic drawing of the experimental setup. The two QDs are located in two cryostats separated by $0.5 \mathrm{~m}$ and held at temperatures between 5 and $15 \mathrm{~K}$. Both QDs are pumped by the same pulsed excitation laser. The emission is dispersed by a high-resolution spectrometer. Photon correlation studies are carried out in a fiber-coupled Hong-Ou-Mandel setup.

asymmetry of the structure allows us to increase the photon outcoupling to values $>10 \%$.

The experimental setup is sketched in Fig. 1. The QD singlephoton sources are mounted in two cryostats on the same optical table with a spatial distance of $0.5 \mathrm{~m}$. Both specimens are excited by the same picosecond-pulsed Ti:sapphire laser with a repetition frequency of $82 \mathrm{MHz}$ (pulse separation: $12.2 \mathrm{~ns}$ ) at a wavelength of $911 \mathrm{~nm}$. Scattered laser light is suppressed with a coarse bandpass filter in the beam path. The beam paths are combined at a polarizing beam splitter (PBS), so that the photons originating from QD1 (QD2) enter the spectrometer (spectral resolution: $30 \mu \mathrm{eV}$ ) in horizontal $\mathrm{H}$ [vertical (V)] polarization. After spectral filtering, the QD emission is coupled into a polarizing fiber splitter to separate the photons again. While the emission of QD1 is sent through a variable optical fiber delay (VOFD) to adjust the time difference between the two photon pulses, the polarization of the photons from QD2 is turned to $\mathrm{H}$ to make the photons indistinguishable in polarization. The TPI takes place at the final 50/50 nonpolarizing fiber beam splitter. For photon autocorrelation measurements, two single-photon counting modules consisting of silicon avalanche singlephoton detectors (APDs) with a timing resolution of nominally $400 \mathrm{ps}$ are connected to the end of the output arms of the final beam splitter.

\section{CHARACTERISTICS OF SINGLE-PHOTON EMISSION}

The emission characteristics of the two QDs, labeled QD1 and QD2 in the following, at a temperature of $4.5 \mathrm{~K}$ under pulsed $p$-shell excitation are shown in Fig. 2. While the quasiresonant excitation scheme leads to well isolated emission lines at $\lambda=930.2 \mathrm{~nm}$ in the photoluminescence spectra depicted in Figs. 2(a) and 2(b), the spectra for wetting layer excitation exhibit multiple emission lines (inset). Singlephoton emission from the two QDs is investigated by performing second-order photon-autocorrelation measurements in a conventional fiber-coupled Hanbury Brown and Twiss setup (not shown in Fig. 1). Figures 2(c) and 2(d) show the results of the autocorrelation measurements recorded from QD1 and QD2. The values $g_{\mathrm{QD} 1}^{(2)}(0)=0.016$ and $g_{\mathrm{QD} 2}^{(2)}(0)=0.046$ are calculated by taking the area of the central peak (from $-6.1 \mathrm{~ns}$ to $+6.1 \mathrm{~ns}$ ) $A_{0}$ divided by the average area of three (a)
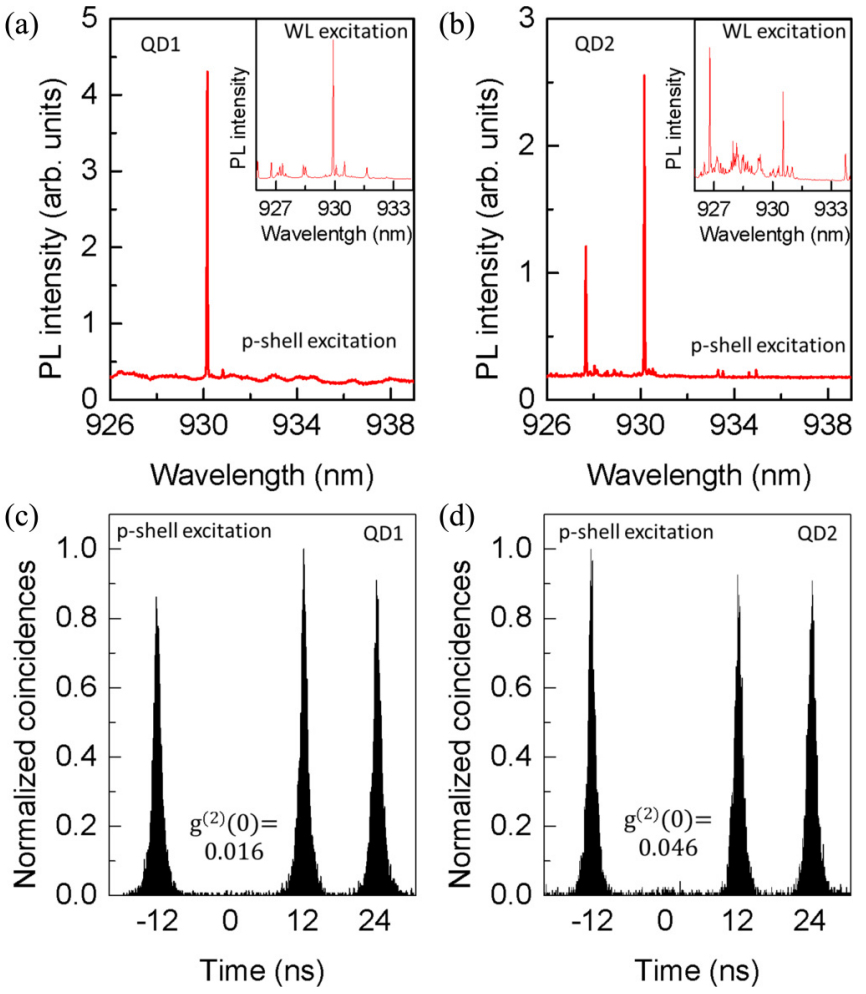

FIG. 2. (Color online) Emission characteristics of QD1/QD2 at a temperature of $T=4.5 \mathrm{~K}$ under pulsed $p$-shell excitation. (a), (b) Photoluminescence spectra (inset: excitation into the wetting layer). (c), (d) Photon autocorrelation function of QD1/QD2. Multiphoton events are strongly suppressed which is reflected in very low $g^{(2)}(0)$ values of 0.016 for QD1 and 0.046 for QD2.

side peaks $A_{s}$, and they demonstrate single-photon emission with very high purity.

Ideally, in order to obtain a maximum degree of indistinguishability, Fourier transform-limited sources are required. In such a case, the relation between coherence time $\tau_{c}$ and lifetime of the QD emission $\tau_{r}$, that describes the visibility of TPI, is [19]

$$
v_{\text {max }, \text { hom }}=\frac{\tau_{c}}{2 \tau_{r}}=1 .
$$

We use a Michelson interferometer to extract the coherence time of the QDs under pulsed $p$-shell excitation. The fringe contrast as a function of interferometer path-length difference is shown in Fig. 3(a). From polarization-dependent measurements we identify that the emission from QD1 originates from a neutral exciton and the emission line of QD2 stems from a charged excitonic complex. The fine-structure splitting of the QD1 line results in an oscillatory behavior in the interference fringe contrast (black stars). A fit to the data with the Fourier transform of two Lorentzians yields coherence times of $\tau_{c 1, \mathrm{QD} 1}=330 \mathrm{ps}$ and $\tau_{c 2, \mathrm{QD} 1}=180 \mathrm{ps}$ and a fine-structure splitting of $9.2 \mu \mathrm{eV}$. We select the fine-structure-split component with longer coherence time by polarization filtering for the TPI experiments in the following sections. The interference fringe contrast of QD2 (red circles) shows an exponential decay with a 1/e coherence time of $\tau_{c, \mathrm{QD} 2}=420 \mathrm{ps}$. 
(a)

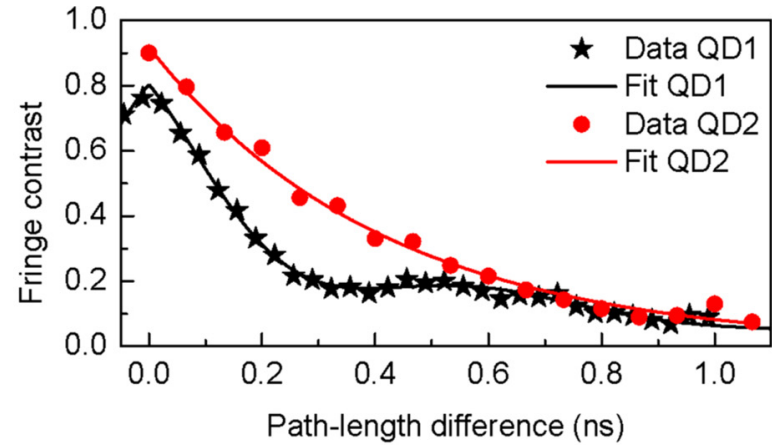

(b)

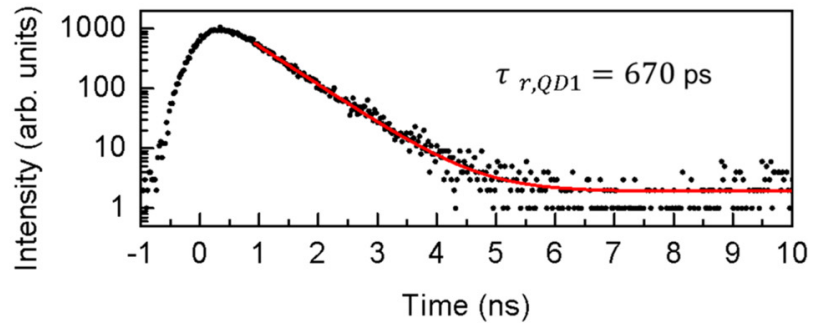

(c)

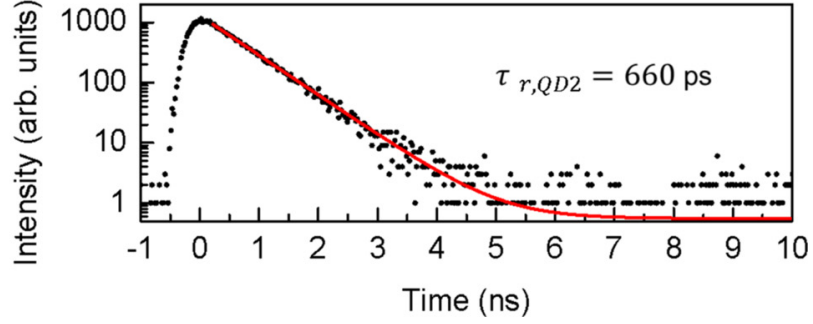

FIG. 3. (Color online) (a) Coherence length measurements for QD1 (black stars) and QD2 (red circles) using a Michelson interferometer. The fine-structure-split exciton lines of QD1 lead to oscillations in the fringe contrast. (b), (c) Time-dependent luminescence of QD1/QD2.

Furthermore, we determine the radiative decay times $\tau_{r}$ of QD1 and QD2 to $\tau_{r, \mathrm{QD} 1}=670 \mathrm{ps}$ and $\tau_{r, \mathrm{QD} 2}=660 \mathrm{ps}$ by fitting an exponential decay according to $I=A e^{-\tau / \tau_{r}}$ to the population decay curves shown in Figs. 3(b) and 3(c), respectively. For both QDs, the emission linewidth is not Fourier transform limited and the maximally expectable visibilities for TPI derived from Eq. (1) would be $v_{\text {max,hom, } \mathrm{QD} 1}=25 \%$ and $v_{\text {max,hom, } \mathrm{QD} 2}=32 \%$.

\section{INTERFERENCE OF CONSECUTIVELY EMITTED PHOTONS FROM THE SAME QD}

In order to probe the emitters' principal performance in photon interference experiments, we study the TPI from consecutive photons emitted from each dot. This experiment is carried out with almost the same experimental setup as shown in Fig. 1, but without the second cryostat and another $50 / 50$ polarization-maintaining fiber beam splitter instead of a polarizing fiber beam splitter. The path-length difference of the second arm of the interferometer is adjusted to the laser repetition period of $12.2 \mathrm{~ns}$, so that two consecutively excited photons can coincide at the same time on the beam splitter. Additionally, there is the possibility to change the time delay $\Delta t$ between the two arms of the interferometer via a variable optical fiber delay.

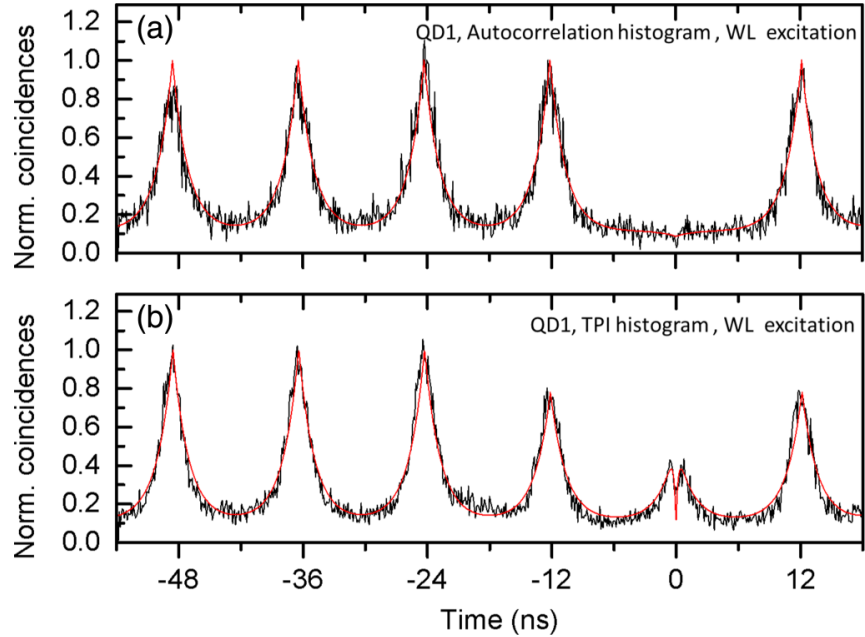

FIG. 4. (Color online) Correlation histograms of QD1 under wetting layer excitation. (a) Photon autocorrelation function. A fit to the data gives a $g^{(2)}(0)$ value of 0.049 . (b) HOM interference of consecutive emitted photons at an interferometer path-length difference of $\Delta t \approx 0$. The red solid curve is a fit to the experimental data (black solid line).

\section{A. Wetting layer excitation}

First, we study the TPI of consecutive emitted photons from QD1 under wetting layer excitation. The autocorrelation histogram of photons emitted from QD1 is shown in Fig. 4(a). As one can see directly from experimental data, the autocorrelation histogram is not as clean as for $p$-shell excitation. Long carrier diffusion lengths lead to a recapturing of charge carriers after a first recombination. In wetting layer excitation this leads to a small constant background at $|\tau|>0$, while in $p$-shell excitation [Figs. 2(c) and 2(d)] this is not visible due to direct excitation of charge carriers into the QDs. From a fit to the data (red solid curve), we extract a $g^{(2)}(0)$ value of $g_{\mathrm{QD} 1, \mathrm{WL}}^{(2)}(0)=0.049$. The corresponding correlation histogram for the TPI of consecutive emitted photons at an interferometer path-length difference of $\Delta t \approx 0$ is shown in Fig. 4(b). Fitting the data with a model based on Ref. [19] yields a nonpostselected visibility of only $v_{\max , \text { hom }}=12 \%$.

\section{B. $p$-shell excitation}

In Figs. 5(a) and 5(b) the second-order correlation function for TPI is shown for zero path-length difference for QD1 and QD2 under $p$-shell excitation. In both cases, the peak at $\tau=0$ is strongly suppressed below a value of 0.5 . The probability for two photons that collide at the beam splitter and exit in opposite directions $g_{\text {indist }}^{(2)}(\tau=0)$ is determined by the area under the peak at $\tau=0$ divided by the averaged area of four peaks for $|\tau|> \pm 12.2 \mathrm{~ns}$. From the raw data we can readily extract values of $g_{\text {indist, } \mathrm{QD} 1}^{(2)}=0.16$ and $g_{\text {indist, }, \mathrm{QD} 2}^{(2)}=$ $0.20<0.5$, verifying the indistinguishability of the photons.

By measuring $g_{\text {indist }}^{(2)}(\tau=0)$ as a function of interferometer path-length offset $\Delta t$, we observe the characteristic HongOu-Mandel dip for $\Delta t=0$. Fitting the data with a twosided exponential $g_{\text {indist }}^{(2)}(\Delta t)=0.5\left[1-v e^{-|\Delta t| / \tau_{m}}\right]$ yields a 

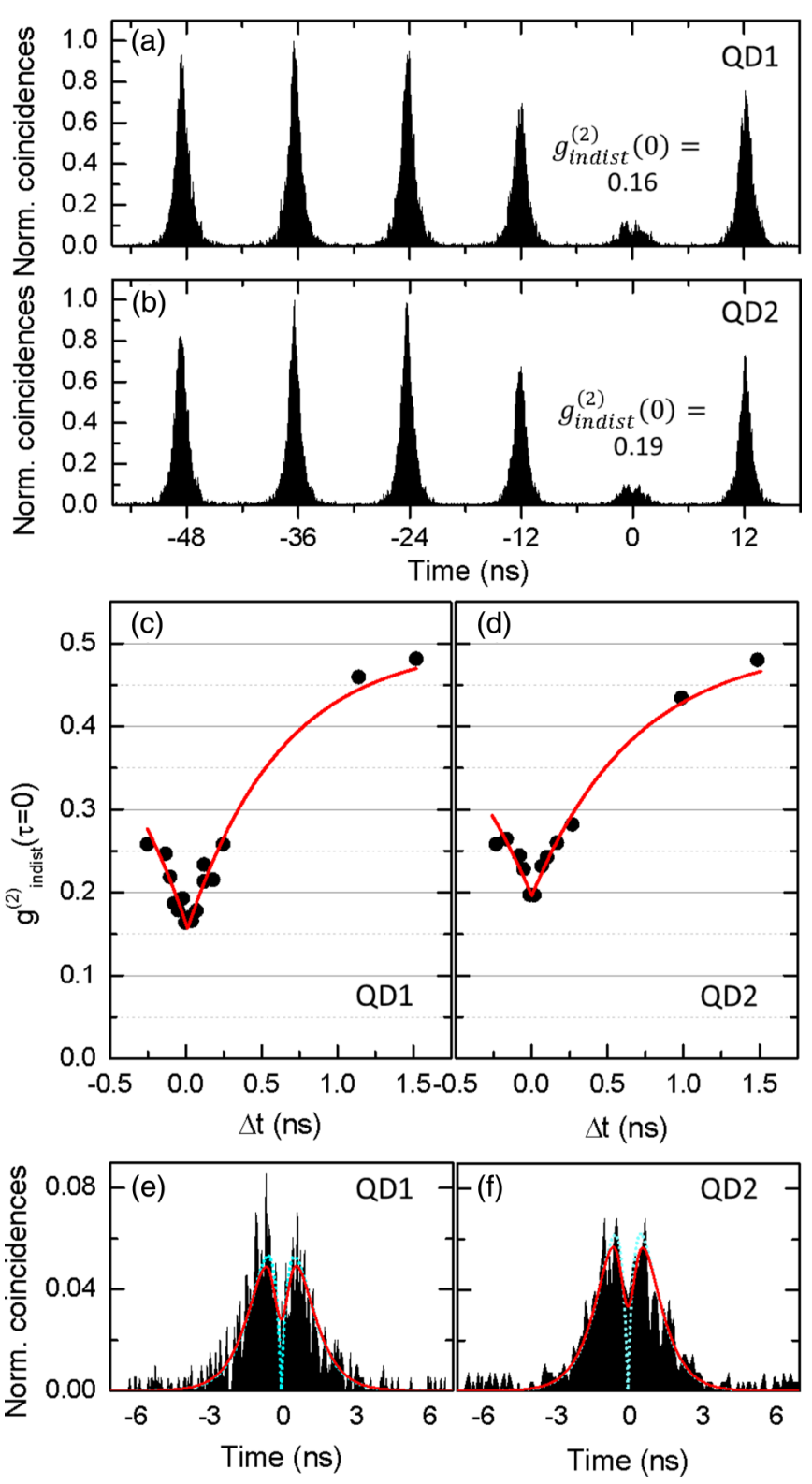

FIG. 5. (Color online) HOM interference of photons from the same QD under $p$-shell excitation. (a), (b) Correlation histogram for emission events from QD1/QD2 at an interferometer path-length difference of $\Delta t \approx 0$. (c), (d) $g_{\text {indist }}^{(2)}(\tau=0)$ as a function of the interferometer path-length offset $\Delta t$ for QD1/QD2. (e), (f) Close-up of the $\tau=0$ peak of (a), (b). The solid red curves are a fit to the data regarding the time response of the detectors, while the dotted blue curves represent the deconvoluted curves without considering any time limitations of the detectors.

nonpostselected value of TPI visibility $v$ of $69 \%$ and $61 \%$ for QD1 [Fig. 5(c)] and QD2 [Fig. 5(d)], respectively. Note that these values are comparable to the values of QDs embedded in micropillar cavities [5,20,21], where the Purcell effect is used to improve $\frac{\tau_{c}}{2 \tau_{c}}$ by reducing the radiative lifetime. For QD1, the visibility under quasiresonant excitation is strongly increased compared to the nonresonant excitation scheme. We attribute this to the lack of recapturing processes and reduced charge carriers in the wetting layer under $p$-shell excitation. For even higher visibilities, strictly resonant excitation is seemingly required [22,23].

A close-up of the $\tau=0$ peak from Figs. 5(a) and 5(b) is shown in Figs. 5(e) and 5(f) for QD1 and QD2, respectively. For both QDs we obtain a dip in the peak at $\tau=0$. For a perfectly balanced beam splitter, an ideal single-photon source, and infinitely fast detectors, this dip would reach zero, which is represented by the dotted blue line. The red solid curve is a fit to the experimental data according to the model in Ref. [19], including the time response of the detectors with $\tau_{c}$ and $\tau_{r}$ being the only fitting parameters. From the fit we extract the coherence time $\tau_{c, \mathrm{QD} 1}=(907 \pm 26) \mathrm{ps}$ and radiative decay time $\tau_{r, \mathrm{QD} 1}=(664 \pm 24) \mathrm{ps}$ of the emission from QD1 $\left(\tau_{c, \mathrm{QD} 2}=(852 \pm 22) \mathrm{ps}\right.$ and $\tau_{r, \mathrm{QD} 2}=(666 \pm 22) \mathrm{ps}$ for QD2 $)$. While the extracted radiative decay times of both QDs fit very well with the time-dependent fluorescence measurements in Fig. 2(a), the coherence times are more than twice as long as measured by the Michelson interferometer. This can be explained by a primary inhomogeneous broadening of the emission lines, for example, by charge fluctuations in the vicinity of the $\mathrm{QD}$, which takes place on a time scale much longer than $12.2 \mathrm{~ns}$ [5]. In this case two consecutive photons have most probably the same emission energy, while photons with larger time spacing differ slightly in energy.

\section{INTERFERENCE OF PHOTONS EMITTED FROM SEPARATE QDS}

For the TPI of separate sources, it is important to match the transition energy of the two QD excitons very precisely. In our system, the emission energies of the two selected QD excitons only differ by $3 \mu \mathrm{eV}$, which allows us to achieve spectral resonance between them by gently readjusting the sample temperature by about $1.8 \mathrm{~K}$ [24]. For accurate data acquisition, it is important to keep the photon counting numbers of both QDs at the same level for the whole integration time. This was done by attenuating the brighter QD's emission to the intensity of the darker one. The striking similarity of the two QDs furthermore allowed us to excite both quantum emitters simultaneously with the same excitation laser into the $p$ shell, which is located $29 \mathrm{meV}$ above the ground-state energy.

The second-order correlation function for TPI from separate sources is shown in Fig. 6(a) for QD1 at $5.00 \mathrm{~K}$ and QD2 at $6.63 \mathrm{~K}$. We determine the opposite output probability
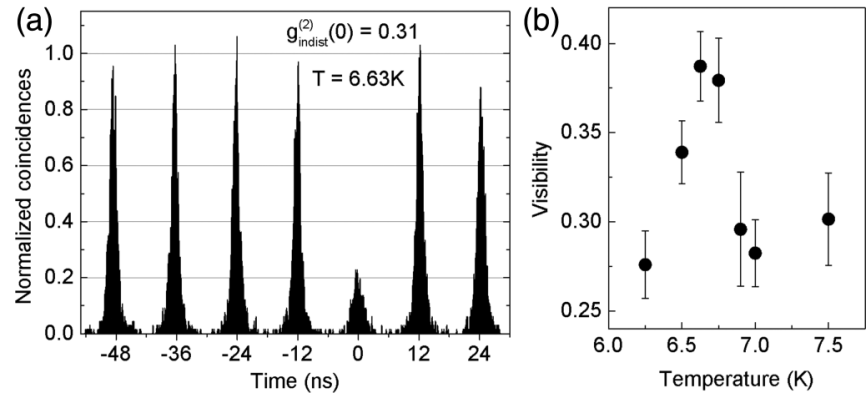

FIG. 6. Results of TPI from two separate sources. (a) Secondorder correlation function for QD1 at $T=5.00 \mathrm{~K}$ and QD2 at $T=6.63 \mathrm{~K}$. (b) TPI visibility as a function of temperature of QD2. 
$g_{\text {indist }}^{(2)}(\tau=0)$ from the raw data by the area of the peak at $\tau=0$ divided by the averaged area of six peaks for $|\tau|>0$. From the data we extract $g_{\text {indist }}^{(2)}(\tau=0)=(0.31 \pm 0.01)$, which verifies that the photons from the two QDs have a nonzero coalescence probability. We calculate the experimental visibility of TPI by $v_{\text {expt }}=\left[0.5-g_{\text {indist }}^{(2)}(\tau=0)\right] / 0.5$, resulting in a value of $v_{\text {expt }}=(39 \pm 2) \%$, exceeding the previously published value of nonresonant TPI experiment of $18.1 \%$ [17] by more than a factor of 2 .

The measured visibility is larger than the visibilities expected from the data in Fig. $3(25 \%-32 \%)$. This cannot be explained by "long" time spectral diffusion, because the time scale on which the broadening occurs does not matter for the TPI of separate sources. Nevertheless, the inhomogeneous broadening has some influence on the TPI visibilities. The interference probability of Fourier transform-limited photons from two independent streams of photons, each fluctuating around a common center frequency according to a Gaussian frequency distribution, is given by (see Appendix)

$$
\begin{aligned}
v_{\text {inhom }} & =1-\frac{2}{T^{2}} \int_{-\infty}^{\infty} d \tau P_{\text {inhom }} \\
& =1-2 \frac{2 \tau_{r} \sigma_{\mathrm{g}}-e^{\frac{1}{4 \tau_{r}^{2} \sigma_{\mathrm{g}}^{2}}} \sqrt{\pi} \operatorname{erfc}\left(\frac{1}{2 \tau_{r} \sigma_{\mathrm{g}}}\right)}{2 \tau_{r} \sigma_{\mathrm{g}}},
\end{aligned}
$$

where $\sigma_{\mathrm{g}}=\sqrt{\sigma_{1}^{2}+\sigma_{2}^{2}}$ and $\sigma_{1,2}^{2}$ are the variance of the frequency distribution for emission from QD1/2. According to the coherence times measured by the Michelson interferometer, this analysis yields a theoretical visibility of $v_{\text {max } \text {,inhom }}=(36.4 \pm 1.5) \%$, which quantitatively explains the high coalescence probability of our sources. We note that very recently a similar analysis has been carried out in Ref. [15] on strictly resonantly driven QDs in the presence of time filtering.

Furthermore, we study the TPI visibility as a function of the temperature of QD2 [Fig. 6(b)]. $v_{\text {expt }}$ shows a maximum at a temperature around $6.7 \mathrm{~K}$. By varying the temperature, the energy difference of the photons rises, resulting in a decrease of TPI visibility.

\section{CONCLUSION}

In conclusion, we have quantitatively analyzed the influence of the excitation scheme on the indistinguishability of single photons emitted from either a single QD or two separate QDs. For the single QD, we found that carrier recapturing can severely spoil the interference visibility of nonpostselected photons in nonresonant excitation schemes. In a quasiresonant experiment, we observed TPI visibilities which significantly exceed the expected value $\left(\frac{\tau_{c}}{2 \tau_{r}}\right)$ both in the two-photon interference from a single QD as well as from two separate QDs. While the first scenario can be explained by long-term energy fluctuations of the excitons, the large interference from separate QDs can still be understood by treating each QD as an inhomogeneously broadened band of emitters close to the Fourier limit. We believe that this work represents an important step towards a deeper understanding of photon interference experiments in solid-state systems, where luminescence signals from single emitters easily are subject to inhomogeneous broadening, blinking, and spectral diffusion, even in strictly resonant excitation schemes.

\section{ACKNOWLEDGMENTS}

The authors gratefully thank T. Steinl for expert sample preparation and J. Schary for technical assistance. This work was financially supported by the German Ministry of Education and Research (BMBF) via the project QuaHL-Rep and by the State of Bavaria.

\section{APPENDIX}

In order to describe the TPI visibility from separate singlephoton sources, we adapted a theory from atomic physics to our solid-state system [25]. We assume that the two photons which interfere at the beam splitter originate from independent ensembles of Fourier transform-limited photons. For both ensembles, an inhomogeneous broadening is considered by a Gaussian frequency distribution which effectively reduces the two-photon interference visibility in HOM experiments.

\section{Single-photon wave packets}

Single-photon wave packets are described by their Fouriertransformed emission spectrum $\Phi(\omega)$,

$$
\xi_{i=1,2}(t)=\int_{-\infty}^{\infty} d \omega \Phi_{i}(\omega) e^{-i \omega t} .
$$

For QDs the single-photon wave packets are one-sided exponential functions in time domain [26]

$$
\begin{aligned}
& \xi_{1}(t)= \begin{cases}\sqrt[4]{\frac{1}{\pi \tau_{r}}} e^{-\frac{t-\frac{\delta \tau}{2}}{2 \tau_{r}}-i\left(\omega-\frac{\Delta}{2}\right) t} & \text { for } t-\frac{\delta \tau}{2}>0 \\
0 & \text { otherwise }\end{cases} \\
& \xi_{2}(t)= \begin{cases}\sqrt[4]{\frac{1}{\pi \tau_{r}}} e^{-\frac{t+\frac{\delta \tau}{2}}{2 \tau_{r}}-i\left(\omega+\frac{\Delta}{2}\right) t} & \text { for } t+\frac{\delta \tau}{2}>0 \\
0 & \text { otherwise }\end{cases}
\end{aligned}
$$

with $\delta \tau$ being the time difference between the two wave packets, $\Delta$ the frequency difference, and $\tau_{r}$ the radiative decay time of the excitonic transition.

\section{Correlation function}

The correlation function for coincidence events at the outputs (channels 3 and 4) of the beam splitter is

$$
G_{3,4}^{(2)}\left(t_{0}, t_{0}+\tau\right)=\operatorname{Tr}\left(\hat{\rho}_{1,2} \hat{A}_{3,4}\right)
$$

with $\hat{\rho}_{1,2}$ describing the two-photon input state and $\hat{A}_{3,4}=$ $\hat{a}_{3}^{\dagger}\left(t_{0}\right) \hat{a}_{4}^{\dagger}\left(t_{0}+\tau\right) \hat{a}_{4}\left(t_{0}\right) \hat{a}_{3}\left(t_{0}+\tau\right)$. The correlation function for two Fourier transform-limited photons with the same polarization is given by [25]

$G_{\mathrm{TL}}^{(2)}\left(t_{0}, t_{0}+\tau\right)=\frac{\left|\xi_{1}\left(t_{0}\right) \xi_{2}\left(t_{0}+\tau\right)-\xi_{2}\left(t_{0}\right) \xi_{1}\left(t_{0}+\tau\right)\right|^{2}}{4}$

The probability for detecting a photon at time $t_{0}+\tau$ in one output of the beam splitter while a photon is detected at time $t_{0}$ in the other one is given by [25]

$$
P_{\mathrm{TL}}=\eta_{3} \eta_{4} T^{2} \int_{-\infty}^{\infty} d t_{0} G_{\mathrm{TL}}^{(2)}\left(t_{0}, t_{0}+\tau\right),
$$


with $\eta_{3,4}$ being the detector efficiencies, which are set to $\eta_{3,4}=1$ in the following, and $T$ being the detector time resolution. For parallel polarized photons this leads to [26]

$$
P_{\mathrm{TL}}\left(\tau, \delta \tau, \tau_{r}, \Delta\right)=\frac{T^{2}}{8 \tau_{r}}\left(e^{-\frac{|\delta \tau-\tau|}{\tau_{r}}}+e^{-\frac{|\delta \tau+\tau|}{\tau_{r}}}-2 \cos (\Delta \tau) e^{-\frac{|\delta \tau|+|\tau|}{\tau_{r}}}\right)
$$

\section{Frequency jitter}

In the case of semiconductor-based single-photon emitters, the mode function $\Phi(\omega)$ of the photons is subject to small variations in frequency, for example, by coupling to phonons, or charge and spin noise [27]. Here, we only assume inhomogeneous broadening, which is represented by a Gaussian frequency distribution $f(\omega)$ with $\sigma^{2}$ being the variance:

$$
f_{i=1,2}\left(\omega_{i}\right)=\frac{1}{\sqrt{\pi} 2 \sigma_{i}} e^{-\frac{\left(\omega_{0 i}-\omega_{i}\right)^{2}}{2 \sigma_{i}^{2}}} .
$$

In this case, the state is given by the quantum mechanical density operator

$$
\hat{\rho}=\int d \omega f(\omega) \hat{\rho}[\xi(\omega)],
$$

with $\hat{\rho}[\xi(\omega)]=\left|1_{\xi}\right\rangle\left\langle 1_{\xi}\right|$ being the density operator of pure states. For $\omega_{1}=\omega$ and $\omega_{2}=\omega+\Delta$ we get the frequency distribution of the frequency difference $\Delta$,

$$
f(\Delta)=\int d \Delta f_{1}(\omega) f_{2}(\omega, \Delta)=\frac{1}{\sqrt{\pi} 2 \sigma_{g}} e^{-\frac{\left(\Delta-\Delta_{0}\right)^{2}}{4 \sigma_{g}^{2}}},
$$

with $\Delta_{0}=\omega_{02}-\omega_{01}$ being the difference of averaged center frequencies, i.e., the spectral detuning, of both photon ensembles and $\sigma_{g}=\sqrt{\sigma_{1}^{2}+\sigma_{2}^{2}}$. We assume that the two photons which collide at the beam splitter originate each from independent photon ensemble with density operators $\hat{\rho}_{1}$ and $\hat{\rho}_{2}$, i.e.,

$$
\hat{\rho}_{12}=\hat{\rho}_{1} \otimes \hat{\rho}_{2}=\int d \Delta f(\Delta) \hat{\rho}\left(\xi_{1}, \xi_{2}\right) .
$$

The corresponding correlation function is given by

$$
G_{\text {inhom }}^{(2)}\left(t_{0}, t_{0}+\tau\right)=\int d \Delta f(\Delta) \operatorname{Tr}\left[\hat{\rho}\left(\xi_{1}, \xi_{2}\right) \hat{A}\left(t_{0}, t_{0}+\tau\right)\right] .
$$

with $\operatorname{Tr}\left[\hat{\rho}\left(\xi_{1}, \xi_{2}\right) \hat{A}\left(t_{0}, t_{0}+\tau\right)\right]$ being the correlation function for pure states given by Eq. (A1).

The resulting probability for detecting a photon at time $t_{0}+\tau$ in one output of the beam splitter while a photon is detected at time $t_{0}$ in the other one for inhomogeneous broadened ensembles of photons is given by

$$
\begin{aligned}
P_{\text {inhom }} & =\int_{-\infty}^{\infty} d t_{0} G_{\text {inhom }}^{(2)}\left(t_{0}, t_{0}+\tau\right) \\
& =\int d \Delta f(\Delta) P_{\mathrm{TL}}\left(\tau, \delta \tau, \tau_{r}, \Delta\right) \\
& =\frac{T^{2}}{8 \tau_{r}}\left[e^{-\frac{|\delta \tau-\tau|}{\tau_{r}}}+e^{-\frac{|\delta \tau+\tau|}{\tau_{r}}}-2 \cos \left(\Delta_{0} \tau\right) e^{-\frac{|\delta \tau|+|\tau|}{\tau_{r}}} e^{-\sigma^{2} \tau^{2}}\right] .
\end{aligned}
$$

We are now in a position to calculate the visibility of TPI from two inhomogeneously broadened distributions of single photons for $\delta \tau=0$ and $\Delta_{0}=0$ by

$$
\begin{aligned}
v_{\text {inhom }} & =1-\frac{2}{T^{2}} \int_{-\infty}^{\infty} d \tau P_{\text {inhom }} \\
& =1-2 \frac{2 \tau_{r} \sigma_{\mathrm{g}}-e^{\frac{1}{4 \tau_{r}^{2} \sigma_{\mathrm{g}}^{2}}} \sqrt{\pi} \operatorname{erfc}\left(\frac{1}{2 \tau_{r} \sigma_{\mathrm{g}}}\right)}{2 \tau_{r} \sigma_{\mathrm{g}}} .
\end{aligned}
$$

The spectrum of the Fourier-limited photons has a Lorentzian line shape with a full width at half maximum (FWHM) of $\Gamma_{1}=\frac{1}{\tau_{r}}$. An additional inhomogeneous broadening represented by a Gaussian frequency distribution [Eq. (A2)] results in a Voigt profile with a FWHM of $\Gamma_{V}=$ $0.535 \Gamma_{1}+\sqrt{0.217 \Gamma_{1}^{2}+\Gamma_{G}^{2}}$ [28], where $\Gamma_{G}=2 \sqrt{2 \ln 2} \sigma_{i}$. This advanced description of TPI allows us to quantitatively describe our experimental observations. Experimentally we determine $\Gamma_{V}=\frac{2}{\tau_{c}}$ from the coherence time measured with the Michelson interferometer. From $\Gamma_{V}$, we back calculate to $\sigma_{1}$ and $\sigma_{2}$, resulting in a visibility of $v_{\max , \text { inhom }}=(36.4 \pm 1.5) \%$. The error is due to the uncertainty in determining the coherence times.
[1] J.-W. Pan, Z.-B. Chen, C.-Y. Lu, H. Weinfurter, A. Zeilinger, and M. Żukowski, Rev. Mod. Phys. 84, 777 (2012).

[2] P. Kok, W. J. Munro, K. Nemoto, T. C. Ralph, J. P. Dowling, and G. J. Milburn, Rev. Mod. Phys. 79, 135 (2007).

[3] J. L. O’Brien, Science 318, 1567 (2007).

[4] P. Michler, A. Kiraz, C. Becher, W. V. Schoenfeld, P. M. Petroff, Lidong Zhang, E. Hu, and A. Imamoglu, Science 290, 2282 (2000).

[5] C. Santori, D. Fattal, J. Vučković, G. S. Solomon, and Y. Yamamoto, Nature 419, 594 (2002).

[6] W. L. Barnes, G. Björk, J. M. Gérard, P. Jonsson, J. A. E. Wasey, P. T. Worthing, and V. Zwiller, Eur. Phys. J. D 18, 197 (2002).

[7] S. Strauf, N. G. Stoltz, M. T. Rakher, L. A. Coldren, P. M. Petroff, and D. Bouwmeester, Nat. Photon. 1, 704 (2007).
[8] T. Heindel, C. Schneider, M. Lermer, S. H. Kwon, T. Braun, S. Reitzenstein, S. Höfling, M. Kamp, and A. Forchel, Appl. Phys. Lett. 96, 011107 (2010).

[9] D. J. P. Ellis, A. J. Bennett, S. J. Dewhurst, C. A. Nicoll, D. A. Ritchie, and A. J. Shields, New J. Phys. 10, 043035 (2008).

[10] J. M. Gérard, in Semiconductor Quantum Dots, edited by P. Michler (Springer, Berlin, 2003).

[11] H.-J. Briegel, W. Dür, J. I. Cirac, and P. Zoller, Phys. Rev. Lett. 81, 5932 (1998).

[12] J. Hofmann, M. Krug, N. Ortegel, L. Gérard, M. Weber, W. Rosenfeld, and H. Weinfurter, Science 337, 72 (2012).

[13] J. I. Cirac, P. Zoller, H. J. Kimble, and H. Mabuchi, Phys. Rev. Lett. 78, 3221 (1997). 
[14] J. Nilsson, R. M. Stevenson, K. H. A. Chan, J. Skiba-Szymanska, M. Lucamarini, M. B. Ward, A. J. Bennett, C. L. Salter, I. Farrer, D. A. Ritchie, and A. J. Shields, Nat. Photon. 7, 311 (2013).

[15] W. B. Gao, P. Fallahi, E. Togan, A. Delteil, Y. S. Chin, J. MiguelSanchez, and A. Imamoglu, Nat. Commun. 4, 2744 (2013).

[16] R. B. Patel, A. J. Bennett, I. Farrer, C. A. Nicoll, D. A. Ritchie, and A. J. Shields, Nat. Photon. 4, 632 (2010).

[17] E. B. Flagg, A. Muller, S. V. Polyakov, A. Ling, A. Migdall, and G. S. Solomon, Phys. Rev. Lett. 104, 137401 (2010).

[18] K. Konthasinghe, M. Peiris, Y. Yu, M. F. Li, J. F. He, L. J. Wang, H. Q. Ni, Z. C. Niu, C. K. Shih, and A. Muller, Phys. Rev. Lett. 109, 267402 (2012).

[19] J. Bylander, I. Robert-Philip, and I. Abram, Eur. Phys. J. D 22, 295 (2003).

[20] S. Varoutsis, S. Laurent, P. Kramper, A. Lemaître, I. Sagnes, I. Robert-Philip, and I. Abram, Phys. Rev. B 72, 041303 (2005).

[21] O. Gazzano, S. Michaelis de Vasconcellos, C. Arnold, A. Nowak, E. Galopin, I. Sagnes, L. Lanco, A. Lemaître, and P. Senellart, Nat. Commun. 4, 1425 (2013).
[22] Y. M. He, Y. He, Y.-J. Wei, D. Wu, M. Atatüre, C. Schneider, S. Höfling, M. Kamp, Chao-Yang Lu, and J. W. Pan, Nat. Nanotech. 8, 213 (2013).

[23] S. Ates, S. M. Ulrich, S. Reitzenstein, A. Löffler, A. Forchel, and P. Michler, Phys. Rev. Lett. 103, 167402 (2009).

[24] QD1 was beneath one of the first ten apertures we studied. We checked around 1000 further apertures before we found the second QD. The time for finding QD2 was on the order of carrying out all of the correlation measurements of the temperature series shown in Fig. 6(b), $\sim 20 \mathrm{~h}$.

[25] T. Legero, T. Wilk, A. Kuhn, and G. Rempe, Adv. At. Mol. Opt. Phys. 53, 253 (2006).

[26] R. B. Patel, A. J. Bennett, K. Cooper, P. Atkinson, C. A. Nicoll, D. A. Ritchie, and A. J. Shields, Nanotechnology 21, 274011 (2010).

[27] A. V. Kuhlmann, J. Houel, A. Ludwig, L. Greuter, D. Reuter, A. D. Wieck, M. Poggio, and R. J. Warburton, Nat. Phys. 9, 570 (2013).

[28] J. J. Olivero and R. L. Longbothum, J. Quantum Spectrosc. Radiat. Transfer 17, 233 (1977). 